\title{
Markerless Liver Tumor Localization Using Internal Liver Volume Delineated By Four- Dimensional Cone-Beam CT
}

\author{
Naohisa Mizukami ${ }^{1}$, Kiyoshi Yoda ${ }^{2}$ \\ 1. Department of Radiation Oncology, Yame General Hospital, Yame, JPN 2. Research Physics, Elekta K.K., Tokyo, JPN
}

Corresponding author: Naohisa Mizukami, mizumizu196933@yahoo.co.jp

\begin{abstract}
Markerless liver tumor localization has been proposed using an internal liver volume delineated by fourdimensional cone-beam CT (4D CBCT). Liver CT was performed under mid-ventilation breath hold, and transferred to a treatment planning system (TPS) to contour the gross target volume (GTV). Subsequently, liver 4D CBCT was performed and transferred to the TPS. After bone matching between the CT and the 4D $\mathrm{CBCT}$, an internal liver volume was delineated on the liver CT volume as the union of liver volumes within a breathing cycle of the $4 \mathrm{D}$ CBCT volumes. Then, inhale liver volume was delineated on the $4 \mathrm{D}$ CBCT. Next, the internal target volume was defined by expanding the GTV by referring to the liver movement within the respiratory cycle of the $4 \mathrm{D} \mathrm{CBCT}$. Subsequently, all the delineated structures were transferred to the 4D CBCT unit. Immediately before treatment, $4 \mathrm{D}$ CBCT was performed again and the couch was repositioned so that the liver may move superiorly to the internal liver volume boundary and inferiorly to the inhale liver volume boundary during the respiratory cycle. The target localization accuracy of the proposed method was evaluated by comparing it to a published lipiodol-based technique. Both methods were applied to a single case in which lipiodol remained inside the tumor. 3D couch repositioning vectors for the two procedures were collected for 25 fraction data of the above same patient, and the differences in the vectors were calculated. The target localization deviations of the proposed method in reference to the lipiodol-based procedure were $0.7 \mathrm{~mm} \pm 0.9 \mathrm{~mm}$ (SD) in the lateral direction, $2.0 \mathrm{~mm} \pm 0.7 \mathrm{~mm}$ (SD) in the superior-inferior direction, and $-2.1 \mathrm{~mm} \pm 0.8 \mathrm{~mm}$ (SD) in the anterior-posterior direction. Markerless liver tumor localization is feasible by delineating the internal liver volume and the inhale liver volume using $4 \mathrm{D} \mathrm{CBCT.}$
\end{abstract}

Review began 03/01/2021 Review ended 04/06/2021 Published 04/13/2021

\section{() Copyright 2021}

Mizukami et al. This is an open access article distributed under the terms of the Creative Commons Attribution License CC-BY 4.0., which permits unrestricted use, distribution, and reproduction in any medium, provided the original author and source are credited.
Categories: Radiation Oncology

Keywords: markerless, liver tumor, localization, four-dimensional cone-beam computed tomography, internal liver volume, $4 \mathrm{~d}$ cbct, lipiodol, image guided radiotherapy, igrt

\section{Introduction}

Lipiodol retention is regarded as a surrogate for cone-beam CT (CBCT) image guidance in radiotherapy of a liver tumor [1-3], allowing the tumor-to-tumor registration between planning and treatment stages. A limitation is that the lipiodol, which was injected at the time of interventional radiology, may be washed out from the tumor after a while, and it may be unavailable at the time of radiotherapy. This happens frequently to the liver tumor patients in our facility. In addition, lipiodol is available only when transcatheter arterial chemoembolization (TACE) was performed with the patient's consent. A metal marker can also be implanted for liver radiotherapy as an alternative technique. However, the invasive embedding of the marker may lead to serious complications such as bleeding. It is also known that the target localization accuracy decreases when the tumor-to-marker distance increases [4]. Recently, 4D CBCT has been widely employed for target localization for respiratory moving tumors [5]. The purpose of this study is to propose a markerless liver tumor localization using the moving liver volume as a surrogate which is delineated by 4D CBCT. The proposed technique may allow 4D CBCT image-guided radiotherapy for a liver tumor without using an external marker even when lipiodol is unavailable.

\section{Case Presentation}

A 73-year-old female patient was diagnosed with cirrhosis type $\mathrm{C}$, and subsequently diagnosed with hepatocellular carcinoma (HCC) in segment six. TACE was performed twice, resulting in local control failure, and thereafter radiotherapy was ordered. Partial lipiodol retention was observed in the HCC.

Liver CT was performed under mid-ventilation breath hold, and transferred to a treatment planning system (TPS) to contour the gross target volume (GTV). Subsequently, liver 4D CBCT was performed and transferred to the TPS. After bone matching between the CT and the 4D CBCT, an internal liver volume was delineated on the liver CT volume as the union of liver volumes within a breathing cycle of the 4D CBCT volumes, where a concept of internal organ volume was first mentioned by Li et al [6]. Then, inhale liver volume was delineated on the 4D CBCT. Next, the internal target volume was defined by expanding the GTV by referring to the liver movement within the respiratory cycle of the 4D CBCT. Subsequently, all the defined structures were transferred to the $4 \mathrm{D}$ CBCT unit. 
Immediately before treatment, 4D CBCT was performed again and the couch was repositioned so that the liver may move superiorly to the internal liver volume boundary and inferiorly to the inhale liver volume boundary during the respiratory cycle. To reduce the patient's respiratory amplitude, a vacuum-activated immobilization unit, BodyFIX (Elekta, Stockholm, Sweden) was employed.

The target localization accuracy of the proposed method was evaluated by comparing it to the lipiodol-based technique [1-3]. Both methods were applied to the present case in which lipiodol remained inside the tumor. 3D couch repositioning vectors for the two procedures were collected for 25-fraction CBCT data of the above patient, and the differences in the vectors were calculated.

Video 1 shows 4D CBCT images of a liver tumor on coronal (left) and sagittal (right) planes with intratumoral lipiodol retention. In this case, the contours of the tumor and the lipiodol are assumed identical. Immediately before treatment, the patient couch was repositioned so that the moving tumor with lipiodol stayed inside the internal target volume (ITV) in pink during a breathing cycle, where the ITV was defined in a TPS.

\section{VIDEO 1: 4D CBCT images of a liver tumor on coronal (left) and sagittal} (right) planes with intratumoral lipiodol retention.

4D СВСТ: Four-dimensional cone-beam computed tomography.

View video here: https://vimeo.com/516116524

Video 2 demonstrates 4D CBCT images of a liver tumor on coronal (left) and sagittal (right) planes with a delineated internal liver volume (light pink) and an inhale liver volume (yellow). Immediately before treatment, the patient couch was repositioned so that the liver may move superiorly to the internal liver volume boundary and inferiorly to the inhale liver volume boundary during a respiratory cycle.

\section{VIDEO 2: 4D CBCT images of a liver tumor on coronal (left) and sagittal (right) planes with a delineated internal liver volume (light pink) and an inhale liver volume (yellow). \\ 4D СBCT: Four-dimensional cone-beam computed tomography.}

View video here: https://vimeo.com/516118398

The target localization deviations of the proposed method in reference to the lipiodol-based procedure were $0.7 \mathrm{~mm} \pm 0.9 \mathrm{~mm}$ in the lateral direction, $2.0 \mathrm{~mm} \pm 0.7 \mathrm{~mm}$ in the superior-inferior direction, and $-2.1 \mathrm{~mm} \pm$ $0.8 \mathrm{~mm}$ in the anterior-posterior direction.

\section{Discussion}

To the authors' best knowledge, this is the first report proposing a markerless liver tumor localization using an internal liver volume and an inhale liver volume delineated by $4 \mathrm{D} \mathrm{CBCT.} \mathrm{The} \mathrm{major} \mathrm{uncertainty} \mathrm{of} \mathrm{the}$ proposed target localization was $2 \mathrm{~mm}$ in the superior-inferior and the anterior-posterior directions. Our liver treatment planning mostly employs an isotropic margin of $5 \mathrm{~mm}$ [7]. The above uncertainty is therefore clinically acceptable.

Because the means of the localization errors are significantly larger than the SDs in the above two directions, systematic errors are dominant factors. The systematic errors may result from our procedure of registering 4D CBCT and 3D planning CT using bone matching. By employing 4D planning CT, a more accurate registration may be performed. It was also reported that respiratory movement of the liver has rotational components [8], which may bring about some uncertainties in the proposed liver contour matching on the coronal and sagittal planes. However, the advantage of noninvasive liver radiotherapy outweighs the errors observed in this study.

In this case study, the tumor was located in the superior part. We have no data for a tumor in the inferior part, which may need another analysis.

Lastly, the proposed technique may be easily employed and verified in any institution having a linac with 4D CBCT functionality.

\section{Conclusions}

We have proposed a new method for markerless liver tumor localization using an internal liver volume and 
an inhale liver volume delineated by $4 \mathrm{D}$ CBCT. Localization accuracy was measured in reference to the published lipiodol-based technique, leading to an uncertainty of $2 \mathrm{~mm}$ which was considered clinically acceptable.

\section{Additional Information \\ Disclosures}

Human subjects: Consent was obtained or waived by all participants in this study. Conflicts of interest: In compliance with the ICMJE uniform disclosure form, all authors declare the following: Payment/services info: All authors have declared that no financial support was received from any organization for the submitted work. Financial relationships: All authors have declared that they have no financial relationships at present or within the previous three years with any organizations that might have an interest in the submitted work. Other relationships: All authors have declared that there are no other relationships or activities that could appear to have influenced the submitted work.

\section{References}

1. Hawkins MA, Brock KK, Eccles C, Moseley D, Jaffray D, Dawson LA: Assessment of residual error in liver position using $\mathrm{kV}$ cone-beam computed tomography for liver cancer high-precision radiation therapy. Int J Radiat Oncol Biol Phys. 2006, 66:610-619. 10.1016/i.ijrobp.2006.03.026

2. Nakagawa K, Yamashita H, Igaki H, Terahara A, Shiraishi K, Yoda K: Contrast medium-assisted stereotactic image-guided radiotherapy using kilovoltage cone-beam computed tomography. Radiat Med. 2008, 26:570572. 10.1007/s11604-008-0275-2

3. Yue J, Sun X, Cai J, et al.: Lipiodol: a potential direct surrogate for cone-beam computed tomography image guidance in radiotherapy of liver tumor. Int J Radiat Oncol Biol Phys. 2012, 82:834-841. 10.1016/j.ijrobp.2010.12.050

4. Seppenwoolde Y, Wunderink W, Wunderink-van Veen SR, Storchi P, Méndez Romero A, Heijmen BJ: Treatment precision of image-guided liver SBRT using implanted fiducial markers depends on markertumour distance. Phys Med Biol. 2011, 56:5445-5468. 10.1088/0031-9155/56/17/001

5. Sonke J-J, Zijp L, Remeijer P, van Herk M: Respiratory correlated cone beam CT. Med Phys. 2005, 32:11761186. 10.1118/1.1869074

6. Li G, Markova S, Garcia A, et al.: Clinical evaluation of automatic organ contour propagation using 4DMRI images. Int J Radiat Oncol Biol Phys. 2017, 99:E685-E686. 10.1016/j.ijrobp.2017.06.2255

7. Wolthaus JW, Sonke J-J, van Herk M, Belderbos JS, Rossi MM, Lebesque JV, Damen EM: Comparison of different strategies to use four-dimensional computed tomography in treatment planning for lung cancer patients. Int J Radiat Oncol Biol Phys. 2008, 70:1229-1238. 10.1016/j.ijrobp.2007.11.042

8. Xu Q, Hanna G, Grimm J, et al.: Quantifying rigid and nonrigid motion of liver tumors during stereotactic body radiation therapy. Int J Radiat Oncol Biol Phys. 2014, 90:94-101. 10.1016/j.ijrobp.2014.05.007 